\title{
Efficent Synthesis of
}

\section{5-(4-(2,3,4,6-Tetra-O-acetyl- $\beta$-D-glucopyranosyl))benzylidene -2,2-dimethyl-1,3-dioxane-4,6-dione Derivatives}

\author{
Zhou Peng ${ }^{1}$, Xu Zhaohui ${ }^{2, *}$ \\ ${ }^{1}$ Energy and Environment Engineering Institute, Nanchang Institute of Technology, Nanchang, China \\ ${ }^{2}$ Chemistry and Chemical Engineering Department, Jiangxi Normal University, Nanchang, China
}

\section{Email address:}

gotoxzh@163.com (Xu Zhaohui)

${ }^{*}$ Corresponding author

\section{To cite this article:}

Zhou Peng, Xu Zhaohui. Efficent Synthesis of

5-(4-(2,3,4,6-Tetra-O-acetyl- $\beta$-D-glucopyranosyl))benzylidene-2,2-dimethyl-1,3-dioxane-4,6-dione Derivatives. Science Discovery.

Vol. 5, No. 7, 2017, pp. 519-523. doi: 10.11648/j.sd.20170507.18

Received: October 23, 2017; Accepted: November 6, 2017; Published: December 28, 2017

\begin{abstract}
Objective Synthesis of gastrodin intermediate 4-formylphenyl(2,3,4,6-O-tetraacetyl)- $\beta$-D-glucoside analogues. Methods 5-(4-(2,3,4,6-Tetra-O-acetyl- $\beta$-D-glucopyranosyl)) benzylidene-2,2-dimethyl-1,3-dioxane-4,6-dione derivatives were synthesized by the Knoevenagel reaction of self-made 4 -formylphenyl(2,3,4,6-O-tetraacetyl)- $\beta$-D-glucoside and 2,2-dimethyl-1,3-dioxane -4,6-dione in the presence of $N$-methylimidazole trifluoromethyl sulfonate. Results and conclusions five products of gastrodin intermediate analogues characterized by NMR and MS(ESI). The reaction has the advantages of high yields $(76 \% \sim 84 \%)$, mild conditions, simple operation and environmental friendliness. $N$-methylimidazole trifluoromethyl sulfonate could be recycled and reused several times without significant loss of its efficiency.
\end{abstract}

Keywords: $N$-methylimidazole Trifluoromethyl Sulfonate, 2,2-dimethyl-1,3-dioxane-4,6-dione, Knoevenagel Condensation Reaction, 4-formylphenyl- $\beta$-D-glucoside

\section{5-(4-(2,3,4,6-O-四乙酰基- $\beta$-D-匍萄糖基))苯基亚甲基-2,2-二甲基 $-1,3-$ 二噁烷-4,6-二酮的有效合成}

周鹏 ${ }^{1}$, 许招会 ${ }^{2^{*}}$

1新能源与环境工程学院, 南昌理工学院, 南昌, 中国

2化学化工学院, 江西师范大学, 南昌, 中国

邮箱

gotoxzh@163.com（许招会）

摘要：目的合成天麻素中间体4-甲酰基苯基-2,3,4,6-O-四乙酰基-- $\beta$-D-葡萄糖苷类似物。方法在 $\mathrm{N}$-甲基咪唑三氟甲基磺 酸盐催化作用下，以自制的4-甲酰基苯基-2,3,4,6-O-四乙酰基- $\beta$-D-葡萄糖苷和 2,2 -二甲基-1,3-二噁烷-4,6-二酮为原料, 发生Knoevenagel缩合反应合成了 5-(4-(2,3,4,6-O-四乙酰基- $\beta$-D-葡萄糖基))苯基亚甲基-2,2-二甲基-1,3-二噁烷-4,6-二酮 衍生物。结果与结论共合成了 5 个天麻素中间体类似物, 并对其结构经NMR和MS(ESI)确证. 该反应具有收率高 $(76 \%$ 84\%)、反应温和、操作简单及环境友好等优点。此外, N-甲基咪唑三氟甲基磺酸盐还可重复使用。 
关键词： N-甲基咪唑三氟甲基磺酸盐，2,2-二甲基-1,3-二啞烷-4,6-二酮，Knoevenagel缩合反应， 4 -甲酰基苯基- $\beta$-D-葡萄糖苷

\section{1. 引言}

失眠是临床上最常见的症状, 发病率非常高。在失 眠患者中近 $25 \%$ 程度相当严重, 失眠严重地影响人们的生 活、工作及身心健康。临床使用的镇静催眠合成药物具 有一定的副作用或药物依赖性, 而多数天然的4-甲酰基芳 基- $\beta$-D-糖苷在治疗失眠障碍时疗效较好, 且不良反应少、 完全性好 $[1,2]$ 。但由于存在作用强度较弱, 给药量较大, 生物利用度较低等不足, 从而限制了其推广应用[3]。因 此, 开展4-甲酰基芳基- $\beta$-D-糖苷的结构修饰及其生物活 性研究备受国内外学者广泛关注[4]。

Knoevenagel缩合反应是形成碳碳双键, 实现碳链增 长的重要方法 [5]。5-亚烃基丙二酸亚异丙酯化合物是合 成含特殊结构天然产物 [6]和杂环化合物 [7]的关键中间 体。有关5-亚烃基丙二酸亚异丙酯的合成报道较多, 主 要有无机碱[8]、有机胺[9]或路易斯酸盐[10,11]催化法, 这些方法存在产品收率较低, 反应时间长、后处理复杂 等不足。在DMF或DMSO溶剂中无催化剂合成5-亚烃基
丙二酸亚异丙酯也有文献报道。该方法后处理复杂, 溶 剂不易回收, 因此发展绿色、简便和高效合成的新方法 显得极为迫切。

以消除环境污染、提高反应效率和原子经济性、降 低能耗为研究目的的绿色化学, 是化学重点发展方向之 一[12]。离子液体作为一种温和、绿色反应介质, 在物质 分离、电化学、催化剂、有机合成、材料制备等领域得 到了广泛的应用 $[13,14]$ 。 $N$-甲基咪唑三氟甲磺酸盐是一种 新型的环境友好型溶剂和液体酸催化剂, 它同时拥有液 体酸的高反应活性和固体酸的不挥发性的优点, 已用于 催化缩醛化反应[15]。基于此,笔者探索了以 $N$-甲基咪唑三 氟甲磺酸盐为催化剂, 通过2,3,4,6-O-四乙酰基-4-甲酰基 苯基- $\beta$-D-葡萄糖苷和 2,2-二甲基-1,3-二啞烷-4,6-二酮的 Knoevenagel缩合反应合成了 5种5-(4-(2,3,4,6-O-四乙酰基 - $\beta$-D-葡萄糖基))苯基亚甲基-2,2-二甲基-1,3-二噁烷-4,6二酮衍生物(Scheme 1), 均为新化合物, 并对其结构经 NMR和MS(ESI)表征。

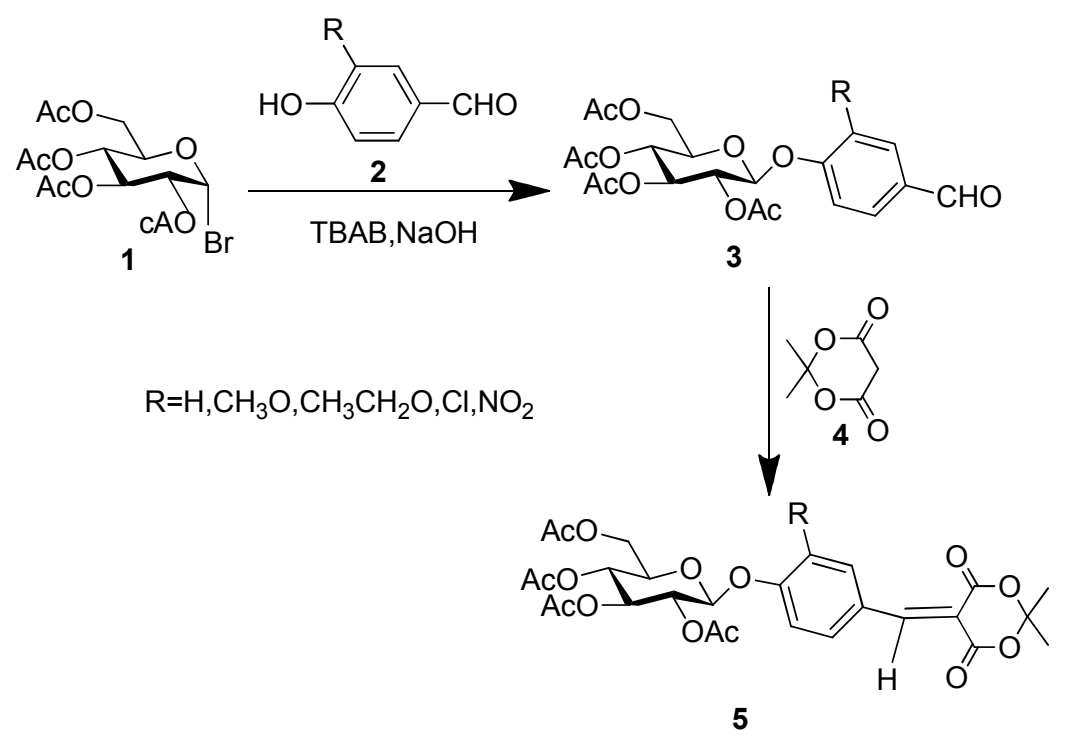

反应式 1 化合物 5 的合成。

\section{2. 结果与讨论}

\section{1. 2,3,4,6-O-四乙酰基-4-甲酰基苯基- $\boldsymbol{\beta}$-D-葡萄糖苷的 合成}

依据参考文献[16,17], 笔者合成了五种4-甲酰基苯 基( $2,3,4,6$-O-四乙酰基)- $\beta$-D-葡萄糖苷衍生物, 其中 $3 \mathrm{~b}$, $3 \mathrm{~d}, 3 \mathrm{e}$ 为新化合物, 实验结果结果见表 1 。从表 1 数据可看 出, 无论是带供电子基或吸电子基对羟基苯甲醛, 反应 都可以顺利进行, 并以 $42 \% \sim 58 \%$ 的较高收率得到相应的
目标产物。对于糖基受体3位取代基对4位-OH有位阻作用, 不利于糖苷化反应, 收率降低。

表1 化合物 $3 \mathrm{a} \sim 3 \mathrm{e}$ 的合成 ${ }^{\mathrm{a}}$

\begin{tabular}{lllll}
\hline Entry & $\mathbf{R}$ & Time(h) & Product & yields (\%) $^{\mathbf{b}}$ \\
\hline 1 & $2 \mathrm{a}(\mathrm{R}=\mathrm{H})$ & 10 & $3 \mathrm{a}$ & 58 \\
2 & $2 \mathrm{~b}\left(\mathrm{R}=3-\mathrm{CH}_{3} \mathrm{O}\right)$ & 8 & $3 \mathrm{~b}$ & 54 \\
3 & $2 \mathrm{c}\left(\mathrm{R}=3-\mathrm{CH}_{3} \mathrm{CH}_{2} \mathrm{O}\right)$ & 8 & $3 \mathrm{c}$ & 55 \\
4 & $2 \mathrm{~d}(\mathrm{R}=3-\mathrm{Cl})$ & 8 & $3 \mathrm{~d}$ & 48 \\
5 & $2 \mathrm{e}\left(\mathrm{R}=3-\mathrm{NO}_{2}\right)$ & 6 & $3 \mathrm{e}$ & 42 \\
\hline \multirow{2}{*}{ reaction conditions: } & 2,3,4,6-O-tetraacetyl- $\alpha$ - $\mathrm{D}$-glucopyranosyl bromide \\
(0.1 mol), 4-hydroxybenzaldhyde derivatives $(0.15$ mol $)$, TBAB (0.002 mol), \\
NaOH solutions $(10 \%, 50 \mathrm{~mL})$, acetone $(60 \mathrm{~mL})$, reaction temperature (r.t.); \\
bisolated yield.
\end{tabular}




\section{2. 反应条件的篮选}

以4-甲酰基苯基( $2,3,4,6-\mathrm{O}$-四乙酰基)- $\beta$ - $\mathrm{D}$-葡萄糖苷 $3 \mathrm{a}$ 和 2,2 -二甲基-1,3-二啞烷-4,6-二酮4为模板反应底物对 反应条件进行了优化, 通过对催化剂、溶剂、温度等条 件的篎选, 确定了较优反应条件,实验结果见表 1 。

初始考察了无催化剂条件下溶剂对反应的影响, 结 果发现在正己烷、乙酸乙酯、水中检测到化合物 $5 \mathrm{a}$ 较少, 在甲醇、乙腈溶剂中化合物 $5 \mathrm{a}$ 收率提高显著, 而以乙醇为
反应介质效果最佳。在乙醇溶剂加入 $20 \mathrm{~mol} \% \mathrm{~N}$-甲基咪唑 三氟甲磺酸盐(MOITf)催化剂, 加热回流 $6 \mathrm{~h}$, 化合物 $5 \mathrm{a}$ 的 收率为 $83 \%$ 。接着考察了催化剂用量、反应温度及反应时 间对产品收率的影响。因此, 较优反应条件为催化剂 MIOTf用量为 $20 \mathrm{~mol} \%$ ， 4-甲酰基苯基(2,3,4,6-O-四乙酰 基)- $\beta$-D-葡萄糖苷与 2,2 -二甲基-1,3-二啞烷-4,6-二酮物质 的量比为 $1.0: 1.2$, 乙醇溶液中回流 $6 \mathrm{~h}$ 。

表2 反应条件的优化 ${ }^{\mathrm{a}}$ 。

\begin{tabular}{|c|c|c|c|c|c|}
\hline Entry & Solvent & Catalyst $/ \mathbf{m o l} \%$ & Time/h & Temperature $/{ }^{\circ} \mathrm{C}$ & Yield $^{\mathrm{b}} / \%$ \\
\hline 1 & n-Hexane & None & 8 & reflux & Trance \\
\hline 2 & EtOAc & None & 8 & reflux & Trance \\
\hline 3 & $\mathrm{CH}_{3} \mathrm{CN}$ & None & 8 & reflux & 56 \\
\hline 4 & $\mathrm{CH}_{3} \mathrm{OH}$ & None & 8 & reflux & 61 \\
\hline 5 & $\mathrm{CH}_{3} \mathrm{CH}_{2} \mathrm{OH}$ & None & 8 & reflux & 68 \\
\hline 6 & $\mathrm{H}_{2} \mathrm{O}$ & None & 10 & 80 & Trance \\
\hline 7 & $\mathrm{CH}_{3} \mathrm{CH}_{2} \mathrm{OH}$ & $N$-methylimidazole trifluoromethyl sulfonate/20 & 6 & reflux & 84 \\
\hline 8 & $\mathrm{CH}_{3} \mathrm{CH}_{2} \mathrm{OH}$ & 1-methylimidazole trifluoroacetate/20 & 8 & reflux & Trance \\
\hline 9 & $\mathrm{CH}_{3} \mathrm{CH}_{2} \mathrm{OH}$ & $N$-methylimidazole trifluoromethyl sulfonate/20 & 10 & 60 & 65 \\
\hline 10 & $\mathrm{CH}_{3} \mathrm{CH}_{2} \mathrm{OH}$ & $N$-methylimidazole trifluoromethyl sulfonate/20 & 7 & reflux & 84 \\
\hline 11 & $\mathrm{CH}_{3} \mathrm{CH}_{2} \mathrm{OH}$ & $N$-methylimidazole trifluoromethyl sulfonate/10 & 6 & reflux & 64 \\
\hline 12 & $\mathrm{CH}_{3} \mathrm{CH}_{2} \mathrm{OH}$ & $N$-methylimidazole trifluoromethyl sulfonate/30 & 6 & reflux & 84 \\
\hline
\end{tabular}

${ }^{a}$ reaction conditions: 4-formylphenyl(2,3,4,6-O-tetraacetyl)- $\beta$-D-glucoside $(1.0 \mathrm{mmol}), 2,2$-pentylene-1,3-dioxane-4,6-dione(1.2 $\left.\mathrm{mmol}\right)$, solvent(5.0 $\left.\mathrm{mL}\right)$; ${ }^{b}$ isolated yield.

\section{3. 反应底物的拓展}

在上述最佳反应条件下，对4-甲酰基苯基(2,3,4,6-O四乙酰基)- $\beta-D$ - 葡萄糖苷反应底物进行了扩展, 合成了五 种 5-(4-(2,3,4,6-O-四乙酰基- $\beta$-D-葡萄糖基))苯基亚甲基 -2,2-二甲基-1,3-二啞烷-4,6-二酮衍生物(5a〜5e)(见表3)。 从表3数据可看出, 无论是带供电子基或吸电子基的4-甲 酰基苯基( $2,3,4,6-O$-四乙酰基)- $\beta$ - $D$ - 葡萄糖苷都较高收率 得到相应的目标产物。

表3 化合物 $5 \mathrm{a} \sim 5 \mathrm{e}$ 的合成 ${ }^{\mathrm{a}}$ 。

\begin{tabular}{lllll}
\hline Entry & $\mathbf{R}$ & Time(h) & Product & yields (\%) \\
\hline 1 & $3 \mathrm{a}(\mathrm{R}=\mathrm{H})$ & 5 & $5 \mathrm{a}$ & 84 \\
2 & $3 \mathrm{~b}\left(\mathrm{R}=2-\mathrm{CH}_{3} \mathrm{O}\right)$ & 8 & $5 \mathrm{~b}$ & 76 \\
3 & $3 \mathrm{c}\left(\mathrm{R}=2-\mathrm{CH}_{3} \mathrm{CH}_{2} \mathrm{O}\right)$ & 8 & $5 \mathrm{c}$ & 78 \\
4 & $3 \mathrm{~d}(\mathrm{R}=2-\mathrm{Cl})$ & 5 & $5 \mathrm{~d}$ & 81 \\
5 & $3 \mathrm{e}\left(\mathrm{R}=2-\mathrm{NO}_{2}\right)$ & 6 & $5 \mathrm{e}$ & 80 \\
\hline
\end{tabular}

${ }^{a}$ reaction conditions: 4-formylphenyl(2,3,4,6-O-tetraacetyl)- $\beta$-D-glucoside (1.0 mmol), 2,2-dimethyl-1,3-dioxane-4,6-dione (1.2 mmol), MOITf (20mol\%), $\mathrm{CH}_{3} \mathrm{CH}_{2} \mathrm{OH}(5.0 \mathrm{~mL})$, reaction temperature (reflux); ${ }^{b}$ isolated yield.

\section{4. 催化剂的回收再利用}

以合成5-(4-(2,3,4,6-O-四乙酰基- $\beta$ - $D$-葡萄糖基))苯基 亚甲基-2,2-二甲基-1,3-二噁烷-4,6-二酮为例, 研究MOITf 的回收再利用。在优化的反应条件下反应结束后, 冷却 至室温, 过滤, 滤液含有MOITf催化剂。按优化反应条件 继续向滤液中投入反应物料。这样重复操作4次, 反应的 收率依次为 $84 \%, 83 \%, 80 \%, 78 \%$ 。可见, MOITf有较 好的催化活性和稳定性。

\section{3. 实验部分}

\section{1. 仪器与试剂}

葡萄糖、4-羟基苯甲醛、3-乙氧基-4-羟基苯甲醛、3甲氧基-4-差基苯甲醛、3-氯-4-羟基苯甲醛及3-硝基-4-羟基 苯甲醛(上海阿拉丁生化科技股份有限公司，分析纯); 其 它试剂均为化学纯, 天津市永大化学试剂有限公司生产。

瑞士BuchiB-540型显微熔点仪(温度计未经校正); 德 国Bruker $400 \mathrm{MHz}$ 型核磁共振仪(DMSO- $d_{6}$ 为溶剂, TMS 为内标); $\mathrm{MS}$ 谱由 $\mathrm{ABI}$ 公司 API3200三重四级杆质谱仪记 录。

\section{2. $\mathbf{N}$-甲基咪唑三氟甲磺酸盐的制备 [18]}

$0 \sim 5^{\circ} \mathrm{C}$ 下将三氟甲磺酸 $(27.2 \mathrm{~mL}, 200.0 \mathrm{mmol})$ 的二氯 甲烷溶液滴加到 $N$-甲基咪唑 $(16.0 \mathrm{~mL}, 200.0 \mathrm{mmol}$ )的干燥 的二氯甲烷 $(30.0 \mathrm{~mL})$ 溶液中, 然后升至室温反应 $2 \mathrm{~h}$, 减 压回收二氯甲烷得粗产品, 再用甲基叔丁基洗涤3次, 真 空干燥得到无色晶体纯品。 ${ }^{1} \mathrm{H}$ NMR (DMSO- $d_{6}, 400 \mathrm{MHz}$ ) $\delta$ : 3.86(s, $3 \mathrm{H}), 7.66 \sim 7.70(\mathrm{~m}, 2 \mathrm{H}), 9.04(\mathrm{~s}, 1 \mathrm{H}), 14.15(\mathrm{~s}$, $1 \mathrm{H}) ;{ }^{13} \mathrm{C}$ NMR (DMSO- $\left.d_{6}, 100 \mathrm{MHz}\right) \delta$ : 35.88, 119.53, $120.19,122.74,123.61,136.26$ 。

\section{3 . 化合物 $\mathbf{3 a} \sim \mathbf{3 e}$ 的合成}

向 $50 \mathrm{~mL}$ 三口瓶中依次加入 4-羟基苯甲醛衍生物 $(0.15 \mathrm{~mol}) 、 10 \%$ 的 $\mathrm{NaOH}$ 溶液 $(50 \mathrm{~mL})$ 、丙酮 $(60 \mathrm{~mL})$ 、相 转移催化剂四丁基溴化铵(TBAB, $0.002 \mathrm{~mol}$ ), 磁力搅拌几 
分钟, 分批加入 $2,3,4,6-\mathrm{O}$-四乙酰基- $\alpha$ - $\mathrm{D}$-溴代葡萄糖 $(0.1$ $\mathrm{mol})$, 室温搅拌6.0 10.0 h。反应毕, 于水浴减压蒸回收 丙酮, 冷却后析出黄色油状物, 倾去水层, 用无水乙醇 适当加热溶解, 冷却, 析出白色固体, 过滤, 室温真空 干燥得目标化合物 $3 \mathrm{a} \sim 3 \mathrm{e}$ 。

4-甲酰基苯基(2,3,4,6-四-O-乙酰基)- $\beta$-D-葡萄糖苷 (3a): 白色固体, 熔点 $142 \sim 143^{\circ} \mathrm{C}$ (lit.[16] $\left.141 \sim 142^{\circ} \mathrm{C}\right) ;{ }^{1} \mathrm{H}$ NMR $\left(400 \mathrm{MHz}, \mathrm{CDCl}_{3}\right) \delta: 2.05(\mathrm{~s}, 3 \mathrm{H}), 2.06(\mathrm{~s}, 6 \mathrm{H})$, $2.07(\mathrm{~s}, 3 \mathrm{H}), 3.94$ (ddd, $J_{1}=7.6 \mathrm{~Hz}, J_{2}=5.2 \mathrm{~Hz}, J_{3}=2.0 \mathrm{~Hz}$, $\left.1 \mathrm{H}, \mathrm{H}^{5}\right), 3.89(\mathrm{~s}, 3 \mathrm{H}), 4.18\left(\mathrm{dd}, J_{1}=12.4 \mathrm{~Hz}, J_{2}=2.0 \mathrm{~Hz}, 1\right.$ $\left.\mathrm{H}, \mathrm{H}^{6 \mathrm{a}}\right), 4.30\left(\mathrm{dd}, J_{l}=12.4 \mathrm{~Hz}, J_{2}=5.2 \mathrm{~Hz}, 1 \mathrm{H}, \mathrm{H}^{6 \mathrm{~b}}\right), 5.16 \sim$ $5.25(\mathrm{~m}, 2 \mathrm{H}), 5.30\left(\mathrm{~d}, J=9.6 \mathrm{~Hz}, 1 \mathrm{H}, \mathrm{H}^{1}\right), 5.32 \sim 5.36(\mathrm{~m}, 1$ H), $7.11(\mathrm{~d}, J=8.8 \mathrm{~Hz}, 2 \mathrm{H}), 7.86(\mathrm{~d}, J=8.8 \mathrm{~Hz}, 2 \mathrm{H}), 9.93(\mathrm{~s}$, $1 \mathrm{H}) ;{ }^{13} \mathrm{C}$ NMR $\left(100 \mathrm{MHz}, \mathrm{CDCl}_{3}\right) \delta: 20.56,20.57,20.58$, $20.63,61.90,68.17,71.05,72.34,72.55,98.09,116.81$, $131.79,131.91,161.24,169.18,169.34,170.14,170.42$, 190.62 。

(4-甲酰基-2-甲氧基)苯基(2,3,4,6-四-O-乙酰基)- $\beta$-D葡萄糖苷(3b): 白色固体, 熔点 $105 \sim 106{ }^{\circ} \mathrm{C} ;{ }^{1} \mathrm{H}$ NMR $(400$ $\left.\mathrm{MHz}, \mathrm{CDCl}_{3}\right) \delta: 2.05(\mathrm{~s}, 6 \mathrm{H}), 2.07(\mathrm{~s}, 3 \mathrm{H}), 2.08(\mathrm{~s}, 3 \mathrm{H})$, $3.86\left(\mathrm{ddd}, J_{l}=7.6 \mathrm{~Hz}, J_{2}=5.2 \mathrm{~Hz}, J_{3}=2.4 \mathrm{~Hz}, 1 \mathrm{H}, \mathrm{H}^{5}\right)$, $4.19\left(\mathrm{dd}, J_{1}=12.4 \mathrm{~Hz}, J_{2}=2.4 \mathrm{~Hz}, 1 \mathrm{H}, \mathrm{H}^{6 \mathrm{a}}\right), 4.28\left(\mathrm{dd}, J_{1}=\right.$ $\left.12.4 \mathrm{~Hz}, J_{2}=5.2 \mathrm{~Hz}, 1 \mathrm{H}, \mathrm{H}^{6 \mathrm{~b}}\right), 5.11 \sim 5.21(\mathrm{~m}, 2 \mathrm{H}), 5.30(\mathrm{~d}$, $\left.J=8.8 \mathrm{~Hz}, 1 \mathrm{H}, \mathrm{H}^{1}\right), 5.32 \sim 5.35(\mathrm{~m}, 1 \mathrm{H}), 7.22(\mathrm{~d}, J=8.0 \mathrm{~Hz}$, $1 \mathrm{H}), 7.41 \sim 7.44(\mathrm{~m}, 2 \mathrm{H}), 9.90(\mathrm{~s}, 1 \mathrm{H}) ;{ }^{13} \mathrm{C}$ NMR $(100 \mathrm{MHz}$, $\left.\mathrm{CDCl}_{3}\right) \delta: 20.56,20.57,20.59,20.65,56.12,61.90,68.29$, $71.06,72.28,72.41,99.74,110.88,118.25,125.29,132.87$, $151.03,151.11,169.20,169.35,170.18,170.45,190.83$; HR-ESI/MS(m/Z) calcd[M] ${ }^{+}=482.1424$, found 482.1416 。

(4-甲酰基-2-乙氧基)苯基(2,3,4,6-四-O-乙酰基)- $\beta$ - $\mathrm{D}$ 葡萄糖苷(3c): 白色固体, 熔点 $115 \sim 116^{\circ} \mathrm{C}$ (lit. [17] 115 $\left.117^{\circ} \mathrm{C}\right) ;{ }^{1} \mathrm{H}$ NMR $\left(400 \mathrm{MHz}, \mathrm{CDCl}_{3}\right) \delta: 1.45(\mathrm{t}, J=6.8 \mathrm{~Hz}, 3$ H), 2.05(s, $6 \mathrm{H}), 2.06(\mathrm{~s}, 3 \mathrm{H}), 2.07(\mathrm{~s}, 3 \mathrm{H}), 3.94\left(\mathrm{ddd}, J_{1}=\right.$ $\left.7.6 \mathrm{~Hz}, J_{2}=5.2 \mathrm{~Hz}, J_{3}=2.4 \mathrm{~Hz}, 1 \mathrm{H}, \mathrm{H}^{5}\right), 4.11(\mathrm{q}, J=6.8 \mathrm{~Hz}$, $2 \mathrm{H}), 4.19\left(\mathrm{dd}, J_{I}=12.4 \mathrm{~Hz}, J_{2}=2.0 \mathrm{~Hz}, 1 \mathrm{H}, \mathrm{H}^{6 \mathrm{a}}\right), 4.28(\mathrm{dd}$, $\left.J_{I}=12.4 \mathrm{~Hz}, J_{2}=5.2 \mathrm{~Hz}, 1 \mathrm{H}, \mathrm{H}^{6 \mathrm{~b}}\right), 5.14 \sim 5.21(\mathrm{~m}, 2 \mathrm{H})$, $5.30\left(\mathrm{~d}, J=9.6 \mathrm{~Hz}, 1 \mathrm{H}, \mathrm{H}^{1}\right), 5.33 \sim 5.37(\mathrm{~m}, 1 \mathrm{H}), 7.21(\mathrm{~d}, J$ $=8.4 \mathrm{~Hz}, 1 \mathrm{H}), 7.40 \sim 7.43(\mathrm{~m}, 2 \mathrm{H}), 9.88(\mathrm{~s}, 1 \mathrm{H}) ;{ }^{13} \mathrm{C} \mathrm{NMR}$ $\left(100 \mathrm{MHz}, \mathrm{CDCl}_{3}\right) \delta: 14.64,20.54,20.56,20.57,20.61$, $61.69,64.70,68.31,71.00,72.19,72.44,99.43,112.19$, $117.99,125.13,132.69,150.13,151.26,169.04,169.34$, $170.14,170.42,190.87$ 。

(4-甲酰基-2-氯)苯基(2,3,4,6-四-O-乙酰基)- $\beta$ - $\mathrm{D}$-葡萄 糖苷(3d): 白色固体, 熔点 $122 \sim 123^{\circ} \mathrm{C} ;{ }^{1} \mathrm{H}$ NMR $(400 \mathrm{MHz}$, $\left.\mathrm{CDCl}_{3}\right) \delta: 2.05(\mathrm{~s}, 3 \mathrm{H}), 2.05(\mathrm{~s}, 3 \mathrm{H}), 2.06(\mathrm{~s}, 3 \mathrm{H}), 2.09(\mathrm{~s}, 6$ $\mathrm{H}), 3.94\left(\mathrm{t}, J=6.4 \mathrm{~Hz}, 1 \mathrm{H}, \mathrm{H}^{5}\right), 4.23(\mathrm{~d}, J=12.4 \mathrm{~Hz}, 1 \mathrm{H}$, $\left.\mathrm{H}^{6 \mathrm{a}}\right), 4.28\left(\mathrm{dd}, J_{l}=12.4 \mathrm{~Hz}, J_{2}=5.2 \mathrm{~Hz}, 1 \mathrm{H}^{\mathrm{H}} \mathrm{H}^{6 \mathrm{~b}}\right), 5.14 \sim$ $5.23(\mathrm{~m}, 2 \mathrm{H}), 5.31\left(\mathrm{~d}, J=9.2 \mathrm{~Hz}, 1 \mathrm{H}, \mathrm{H}^{1}\right), 5.35 \sim 5.42(\mathrm{~m}, 1$ $\mathrm{H}), 7.28(\mathrm{~d}, J=7.6 \mathrm{~Hz}, 1 \mathrm{H}), 7.76(\mathrm{~d}, J=7.6 \mathrm{~Hz}, 1 \mathrm{H})$, 7.92(d, $J=1.2 \mathrm{~Hz}, 1 \mathrm{H}), 9.91(\mathrm{~s}, 1 \mathrm{H}) ;{ }^{13} \mathrm{C}$ NMR $(100 \mathrm{MHz}$, $\left.\mathrm{CDCl}_{3}\right) \delta: 20.62,20.65,20.68,61.83,64.70,68.10,71.59$, $72.25,72.50,99.35,117.16,125.37,129.64,131.77,132.57$, $157.03, \quad 169.12, \quad 169.34, \quad 170.19, \quad 170.46, \quad 189.58$; HR-ESI/MS(m/Z) calcd[M] ${ }^{+}=486.0929$, found 486.0921 .
(4-甲酰基-2-硝基)苯基(2,3,4,6-四-O-乙酰基)- $\beta$ - D-葡 萄糖苷(3e): 白色固体, 熔点 $134 \sim 136{ }^{\circ} \mathrm{C} ;{ }^{1} \mathrm{H}$ NMR (400 $\left.\mathrm{MHz}, \mathrm{CDCl}_{3}\right) \delta: 2.06(\mathrm{~s}, 3 \mathrm{H}), 2.07(\mathrm{~s}, 3 \mathrm{H}), 2.09(\mathrm{~s}, 3 \mathrm{H})$, $2.13(\mathrm{~s}, 3 \mathrm{H}), 3.97$ (ddd, $J_{I}=7.6 \mathrm{~Hz}, J_{2}=4.8 \mathrm{~Hz}, J_{3}=2.8 \mathrm{~Hz}$, $\left.1 \mathrm{H}, \mathrm{H}^{5}\right), 4.22 \sim 4.31\left(\mathrm{~s}, 2 \mathrm{H}, \mathrm{H}^{6 \mathrm{a}}, \mathrm{H}^{6 \mathrm{~b}}\right), 5.18 \sim 5.29(\mathrm{~m}, 2 \mathrm{H})$, $5.31\left(\mathrm{~d}, J=9.2 \mathrm{~Hz}, 1 \mathrm{H}, \mathrm{H}^{1}\right), 5.33 \sim 5.37(\mathrm{~m}, 1 \mathrm{H}),{ }^{13} \mathrm{C} \mathrm{NMR}$ $\left(100 \mathrm{MHz}, \mathrm{CDCl}_{3}\right) \quad \delta$ : 20.49, 20.56, 20.57, 20.66, 61.72, $67.91,70.32,72.06,72.69,99.44,118.93,126.85,131.57$, $134.01,141.31,153.39,169.12,169.27,170.15,170.36$, 188.53; HR-ESI/MS(m/Z) calcd[M] ${ }^{+}=497.1169$, found 497.1183。

\section{4 . 化合物 $\mathbf{5 a} \sim 5 \mathbf{e}$ 的合成}

把 $N$-甲基咪唑三氟甲磺酸盐 $(0.2 \mathrm{mmol})$ 加入到4-甲酰 基苯基(2,3,4,6-四-O-乙酰基)- $\beta$-D-葡萄糖苷 $(1.0 \mathrm{mmol}$ )和 2,2-二甲基-1,3-二噁烷-4,6-二酮 $(1.2 \mathrm{mmol}$ )的乙醇 $(10 \mathrm{~mL}$ ) 溶液中, 搅拌下加热回流5.0 8.0 h, TLC跟踪, 反应完全 后停止加热, 冷却至室温, 过滤, 滤液为含有MOITf的乙 醇溶液并用于下一次反应。滤饼用 $10 \mathrm{~mL}$ 蒸馏水洗涤 2 次、 无水乙醇重结晶、干燥得目标化合物 $5 \mathrm{a} \sim 5 \mathrm{e}$ 。

5 -(4-(2,3,4,6-O-四乙酰基- $\beta$-D-葡萄糖基))苯基亚甲基 -2,2-二甲基-1,3-二噁烷-4,6-二酮 (5a): 淡黄色固体, 熔点 $174 \sim 175^{\circ} \mathrm{C} ;{ }^{1} \mathrm{H}$ NMR $\left(400 \mathrm{MHz}, \mathrm{CDCl}_{3}\right) \delta: 1.80(\mathrm{~s}, 6 \mathrm{H})$, $2.05(\mathrm{~s}, 3 \mathrm{H}), 2.06(\mathrm{~s}, 6 \mathrm{H}), 2.08(\mathrm{~s}, 3 \mathrm{H}), 3.93\left(\mathrm{ddd}, J_{I}=7.6\right.$ $\left.\mathrm{Hz}, J_{2}=5.2 \mathrm{~Hz}, J_{3}=2.4 \mathrm{~Hz}, 1 \mathrm{H}, \mathrm{H}^{5}\right), 4.17\left(\mathrm{dd}, J_{1}=12.4 \mathrm{~Hz}\right.$, $\left.J_{2}=2.4 \mathrm{~Hz}, 1 \mathrm{H}, \mathrm{H}^{6 \mathrm{a}}\right), 4.30\left(\mathrm{dd}, J_{1}=12.4 \mathrm{~Hz}, J_{2}=5.6 \mathrm{~Hz}, 1\right.$ $\left.\mathrm{H}, \mathrm{H}^{6 \mathrm{~b}}\right), 5.16 \sim 5.22(\mathrm{~m}, 2 \mathrm{H}), 5.29 \sim 5.33(\mathrm{~m}, 2 \mathrm{H}), 7.06(\mathrm{~d}, J$ $=8.8 \mathrm{~Hz}, 2 \mathrm{H}), 8.16\left(\mathrm{~d}, J_{I}=8.8 \mathrm{~Hz}, 2 \mathrm{H}\right), 8.38(\mathrm{~s}, 1 \mathrm{H}) ;{ }^{13} \mathrm{C}$ NMR $\left(100 \mathrm{MHz}, \mathrm{CDCl}_{3}\right) \delta: 20.60,20.68,20.70,27.59$, $61.84,68.09,70.95,72.37,72.54,97.91,104.46,112.78$, $116.51,126.77,136.82,157.20,160.14,160.83,163.60$, $169.22,169.37,1170.17,170.50$ 。

5 -(4-(2,3,4,6-O-四乙酰基- $\beta$ - $\mathrm{D}$-葡萄糖基)-3-甲氧基) 苯基亚甲基-2,2-二甲基-1,3-二噁烷-4,6-二酮(5b)：淡黄色 固体, 熔点 $124 \sim 125^{\circ} \mathrm{C} ;{ }^{1} \mathrm{H}$ NMR $\left(400 \mathrm{MHz}, \mathrm{CDCl}_{3}\right) \delta$ : $1.80(\mathrm{~s}, 6 \mathrm{H}), 2.05(\mathrm{~s}, 3 \mathrm{H}), 2.06(\mathrm{~s}, 3 \mathrm{H}), 2.07(\mathrm{~s}, 3 \mathrm{H}), 2.08(\mathrm{~s}$, $3 \mathrm{H}$ ), 3.86 (ddd, $J_{1}=7.6 \mathrm{~Hz}, J_{2}=5.2 \mathrm{~Hz}, J_{3}=2.4 \mathrm{~Hz}, 1 \mathrm{H}$, $\left.\mathrm{H}^{5}\right), 3.89(\mathrm{~s}, 3 \mathrm{H}), 4.19\left(\mathrm{dd}, J_{I}=12.4 \mathrm{~Hz}, J_{2}=2.4 \mathrm{~Hz}, 1 \mathrm{H}\right.$, $\left.\mathrm{H}^{6 \mathrm{a}}\right), 4.28\left(\mathrm{dd}, J_{l}=12.4 \mathrm{~Hz}, J_{2}=5.2 \mathrm{~Hz}, 1 \mathrm{H}, \mathrm{H}^{6 \mathrm{~b}}\right), 5.14 \sim$ $5.20(\mathrm{~m}, 2 \mathrm{H}), 5.29 \sim 5.32(\mathrm{~m}, 2 \mathrm{H}), 7.14(\mathrm{~d}, J=8.8 \mathrm{~Hz}, 1 \mathrm{H})$, $7.57\left(\mathrm{dd}, J_{l}=8.8 \mathrm{~Hz}, J_{2}=2.4 \mathrm{~Hz}, 1 \mathrm{H}\right), 8.17(\mathrm{~d}, J=2.4 \mathrm{~Hz}, 1$ $\mathrm{H}), 8.35(\mathrm{~s}, 1 \mathrm{H}) ;{ }^{13} \mathrm{C}$ NMR $\left(100 \mathrm{MHz}, \mathrm{CDCl}_{3}\right) \delta: 20.58$, $20.61,20.68,27.56,56.14,61.87,68.22,70.98,72.34,72.38$, $99.45,104.44,112.76,117.09,117.45,127.76,130.38$, $149.99,150.92,157.53,160.24,163.67,169.21,169.36$, $170.18,170.49$ 。

5 -(4-(2,3,4,6-O-四乙酰基- $\beta$ - $\mathrm{D}$-葡萄糖基)-3-乙氧基) 苯基亚甲基-2,2-二甲基-1,3-二噁烷-4,6-二酮(5c)：淡黄色 固体, 熔点 $128 \sim 129^{\circ} \mathrm{C} ;{ }^{1} \mathrm{H}$ NMR $\left(400 \mathrm{MHz}, \mathrm{CDCl}_{3}\right) \delta$ : $1.45(\mathrm{t}, J=6.8 \mathrm{~Hz}, 3 \mathrm{H}), 1.80(\mathrm{~s}, 6 \mathrm{H}), 2.05(\mathrm{~s}, 3 \mathrm{H}), 2.06(\mathrm{~s}, 3$ $\mathrm{H}), 2.07(\mathrm{~s}, 3 \mathrm{H}), 2.08(\mathrm{~s}, 3 \mathrm{H}), 3.88\left(\mathrm{ddd}, J_{I}=7.6 \mathrm{~Hz}, J_{2}=5.2\right.$ $\left.\mathrm{Hz}, J_{3}=2.4 \mathrm{~Hz}, 1 \mathrm{H}, \mathrm{H}^{5}\right), 4.11(\mathrm{q}, J=6.8 \mathrm{~Hz}, 2 \mathrm{H}), 4.19\left(\mathrm{dd}, J_{I}\right.$ $\left.=12.4 \mathrm{~Hz}, J_{2}=2.0 \mathrm{~Hz}, 1 \mathrm{H}, \mathrm{H}^{6 \mathrm{a}}\right), 4.28\left(\mathrm{dd}, J_{1}=12.4 \mathrm{~Hz}, J_{2}=\right.$ $\left.5.2 \mathrm{~Hz}, 1 \mathrm{H}, \mathrm{H}^{6 \mathrm{~b}}\right), 5.13 \sim 5.21(\mathrm{~m}, 2 \mathrm{H}), 5.29 \sim 5.38(\mathrm{~m}, 2 \mathrm{H})$, $7.13(\mathrm{~d}, J=8.4 \mathrm{~Hz}, 1 \mathrm{H}), 7.55\left(\mathrm{dd}, J_{l}=8.4 \mathrm{~Hz}, J_{2}=2.0 \mathrm{~Hz}, 1\right.$ 
$\mathrm{H}), 8.15(\mathrm{~d}, J=2.0 \mathrm{~Hz}, 1 \mathrm{H}), 8.34(\mathrm{~s}, 1 \mathrm{H}) ;{ }^{13} \mathrm{C}$ NMR $(100$ $\left.\mathrm{MHz}, \mathrm{CDCl}_{3}\right) \delta: 14.61,20.57,20.60,20.63,20.66,27.54$, $61.87,64.77,68.325,70.93,72.27,72.42,99.24,104.42$ $112.56,117.29,118.25,127.62,130.39,149.17,151.13$ $157.66,160.29,163.71,169.09,169.39,170.21,170.52$ 。

5 -(4-(2,3,4,6-O-四乙酰基- $\beta$ - D- 葡萄糖基)-3-氯)苯基 亚甲基-2,2-二甲基-1,3-二噁烷-4,6-二酮(5d): 淡黄色固体, 熔点 $191 \sim 192^{\circ} \mathrm{C} ;{ }^{1} \mathrm{H}$ NMR $\left(400 \mathrm{MHz}, \mathrm{CDCl}_{3}\right) \delta: 1.80(\mathrm{~s}, 6$ H), 2.05(s, $3 \mathrm{H}), 2.06(\mathrm{~s}, 3 \mathrm{H}), 2.09(\mathrm{~s}, 3 \mathrm{H}), 2.10(\mathrm{~s}, 3 \mathrm{H})$, 3.94(ddd, $\left.J_{l}=7.6 \mathrm{~Hz}, J_{2}=5.2 \mathrm{~Hz}, J_{3}=2.4 \mathrm{~Hz}, 1 \mathrm{H}, \mathrm{H}^{5}\right)$, $4.21\left(\mathrm{dd}, J_{l}=12.4 \mathrm{~Hz}, J_{2}=2.0 \mathrm{~Hz}, 1 \mathrm{H}, \mathrm{H}^{6 \mathrm{a}}\right), 4.30\left(\mathrm{dd}, J_{1}=\right.$ $\left.12.4 \mathrm{~Hz}, J_{2}=5.2 \mathrm{~Hz}, 1 \mathrm{H}, \mathrm{H}^{6 \mathrm{~b}}\right), 5.15 \sim 5.23(\mathrm{~m}, 2 \mathrm{H}), 5.30(\mathrm{t}, J$ $\left.=8.8 \mathrm{~Hz}, 1 \mathrm{H}, \mathrm{H}^{1}\right), 5.38 \sim 5.42(\mathrm{~m}, 1 \mathrm{H}), 7.21(\mathrm{~d}, J=2.4 \mathrm{~Hz}$, $1 \mathrm{H}), 8.03\left(\mathrm{dd}, J_{l}=8.8 \mathrm{~Hz}, J_{2}=2.4 \mathrm{~Hz}, 1 \mathrm{H}\right), 8.23(\mathrm{~d}, J=2.4$ $\mathrm{Hz}, 1 \mathrm{H}), 8.29(\mathrm{~s}, 1 \mathrm{H}) ;{ }^{13} \mathrm{C} \mathrm{NMR}\left(100 \mathrm{MHz}, \mathrm{CDCl}_{3}\right) \delta$ : $20.57,20.61,20.65,27.67,61.78,68.08,70.55,72.25,72.52$, $99.22,104.70,114.06,116.55,124.52,127.69,134.45$, $135.94,155.56,156.35,159.78,163.09,169.09,169.32$, $170.15,170.45$ 。

5 -(4-(2,3,4,6-O-四乙酰基- $\beta$ - D-葡萄糖基)-3-硝基)苯 基亚甲基-2,2-二甲基-1,3-二噁烷-4,6-二酮(5e): 白色固体, 熔点 $195 \sim 197^{\circ} \mathrm{C} ;{ }^{1} \mathrm{H}$ NMR $\left(400 \mathrm{MHz}, \mathrm{CDCl}_{3}\right) \delta$ : $1.81(\mathrm{~s}, 6$ $\mathrm{H}), 2.05(\mathrm{~s}, 3 \mathrm{H}), 2.07(\mathrm{~s}, 3 \mathrm{H}), 2.10(\mathrm{~s}, 3 \mathrm{H}), 2.12(\mathrm{~s}, 3 \mathrm{H})$, $3.96\left(\mathrm{ddd}, J_{l}=7.6 \mathrm{~Hz}, J_{2}=5.2 \mathrm{~Hz}, J_{3}=2.8 \mathrm{~Hz}, 1 \mathrm{H}, \mathrm{H}^{5}\right)$, $4.24\left(\mathrm{dd}, J_{l}=12.4 \mathrm{~Hz}, J_{2}=2.4 \mathrm{~Hz}, 1 \mathrm{H}, \mathrm{H}^{6 \mathrm{a}}\right), 4.29\left(\mathrm{dd}, J_{l}=\right.$ $\left.12.4 \mathrm{~Hz}, J_{2}=5.2 \mathrm{~Hz}, 1 \mathrm{H}, \mathrm{H}^{6 \mathrm{~b}}\right), 5.20(\mathrm{t}, J=9.6 \mathrm{~Hz}, 1 \mathrm{H})$, $5.27 \sim 5.32(\mathrm{~m}, 1 \mathrm{H}), 5.32 \sim 5.37(\mathrm{~m}, 2 \mathrm{H}), 7.39(\mathrm{~d}, J=8.8 \mathrm{~Hz}$, $1 \mathrm{H}), 8.29\left(\mathrm{dd}, J_{1}=8.8 \mathrm{~Hz}, J_{2}=2.4 \mathrm{~Hz}, 1 \mathrm{H}\right), 8.29(\mathrm{~s}, 1 \mathrm{H})$, 8.61(d, $J=2.4 \mathrm{~Hz}, 1 \mathrm{H}) ;{ }^{13} \mathrm{C}$ NMR $\left(100 \mathrm{MHz}, \mathrm{CDCl}_{3}\right) \delta$ : 20.48, 20.56, 20.67, 27.73, 27.75, 61.70, 67.91, 70.30, 72.08, $72.68,99.39,105.05,115.98,118.25,126.95,130.52$, $138.88,140.68,152.42,154.08,159.51,162.55,169.12$, $169.27,170.15,170.41$ 。

\section{4. 结论}

5-(4-(2,3,4,6-O-四乙酰基- $\beta$-D-葡萄糖基))苯基亚甲基 -2,2-二甲基-1,3-二啞烷-4,6-二酮是一种天麻素中间体类 似物。在MOITf的催化作用下, 以乙醇为溶剂, 通过自制 的4-甲酰基苯基 $(2,3,4,6-\mathrm{O}$-四乙酰基)- $\beta$ - $\mathrm{D}$ - 葡萄糖苷和 2,2-二甲基-1,3-二噁烷-4,6-二酮发生Knoevenagel缩合反 应, 有效合成了五种5-(4-(2,3,4,6-O-四乙酰基- $\beta$-D-葡萄糖 基))苯基亚甲基-2,2-二甲基-1,3-二噁烷-4,6-二酮衍生物, 并确定了较好的反应条件：取4-甲酰基苯基(2,3,4,6-O-四 乙酰基)- $\beta$-D-葡萄糖苷 $1.0 \mathrm{mmol}, 2,2$-二甲基-1,3-二啞烷 -4,6-二酮1.2 mmol, MOITf $20 \mathrm{~mol} \%$, 回流反应5.0 8.0 h, 产品收率为 $76 \% \sim 84 \%$ 。该反应具有反应温和、操作简单, 收率较高等优点。此外, MOITf催化剂的回收和重复使用 方便,具有较好的催化活性和稳定性。
致谢

国家科技攻关计划(2001BA323C)和江西省研究生创 新基金项目(YC2015-B023)。

\section{参考文献}

[1] Zhao, J. P.; Pan, W. X.; Chen, W. X. Nat. Prod. Res. Dev. 1992, 4, 35(in Chinese).

[2] Wang, Y. Y.; Tian, L. Y.; Yang, Y.; Sun, W. Y.; Wang, W. Acta Pharmac. Sin. 2017, 52, 378(in Chinese).

[3] Zhu, Q. L.; Li, Y.; Li, J.; Tang, Q.; Guo, C. H.; Yi, S. F. West China J. Pharm. Sci. 2008, 23, 12 (in Chinese).

[4] Bai, Y. F.; Yin, H.; Bi, H. P.; Zhang, Y. B.; Liu, T., Ma, Y. H. Metab. Eng. 2016, 35, 138.

[5] Liu, H. -M.; Liu, H. -L.; Yuan, Y. -J.; Tang, X. R.; Ji, S. J. Chin. J. Org. Chem. 2003, 23, 1159 (in Chinese).

[6] Bruns, R.; Wernicke, A.; Koll, P. Tetrahedron, 1999, 55, 9793.

[7] Huang, Z. -Z.; Wu, L. -L.; Huang, X. Chin. J. Org. Chem. 2000, 20, 88(in Chinese).

[8] Abdallah-EI, A. S.; Texier-Boullet, F.; Hamelin, J. Synthesis 1994, 26, 258.

[9] Shital, S.; Gajanan, R.; Arjun, K.; Santosh, K.; Rajashri, S. Helv. Chim. Acta, 2011, 94, 1943.

[10] Chakrabarty, M.; Mukherjee, R.; Chakrabarty, M.; Arima, S.; Harigaya, Y. Lett. Org. Chem. 2006, 3, 868.

[11] Xu, Z. -H.; Lin, C. -H. Chin. J. Org. Chem. 2013, 33, 1154(in Chinese).

[12] Shi, D. -Q.; Ni, S. -N.; Dou, G. -L. Chin. J. Org. Chem. 2009, 29, 788(in Chinese).

[13] Peter, W.; Wilhelm, K. Angew. Chem., Int. Ed. 2000, 39, 3772 .

[14] Plechkova, N.-V.; Seddon, K.-R. Chem. Soc. Rev. 2008, 370, 123.

[15] Liu, N. -N.; Chen, X.; Zhang, Q. -S.; Wu, Q. P. Chin. J. Org. Chem. 2014, 34, 1364(in Chinese).

[16] Wen, H.; Lin, C. L.; Que, L.; Ge, H.; Ma, L.; Wan, H. Q; Pen, W. L.; Wang, Z. H.; Song, H. C. Eur. J. Med. Chem. 2008, 43, 166.

[17] Lars, K.; Joachim, T. J. Carbohyd. Chem. 2003, 22, 9.

[18] Xu, Z. H.; Zhang, H. F.; Xiong, Y. K.; Liu, D. Y.; Huang, Q. S. Heterocycles, 2016, 92, 2252. 